\title{
Chaotic Transport in Time-Dependent Symplectic Maps
}

\author{
Jicong Shi \\ Department of Physics and Astronomy, The University of Kansas, Lawrence, Kansas 66045-2151
}

(Received 20 October 1995)

\begin{abstract}
The effect of tune modulation in two-dimensional symplectic maps has been studied by using the concept of chaotic transport in terms of flux across resonances. When a single resonance is dominant, the particle escape due to the tune modulation can be characterized by the increment of the flux induced by the modulation. The parameter dependence of the particle escape rate obtained by using the transport theory agrees well with the result of the multiparticle tracking study and the beam study experiments at the CERN Super Proton Synchrotron. This study showed that the transport theory provides a computationally efficient means of studying the particle escape due to the tune modulation and estimating the parameter dependence of the escape rate. [S0031-9007(96)00214-1]
\end{abstract}

PACS numbers: 41.85.-p, 05.45.+b, 29.20.-c

Recent experiments in hadron accelerators have shown that the beam lifetime is substantially decreased when betatron tunes are modulated due to the power supply ripple, ground motion, or synchrobetatron coupling [1,2]. As this phenomenon is not well understood, no effective tool available for estimating the particle loss rate or providing the parameter (modulation amplitude and frequency) dependence of this rate.

In nonlinear dynamics, the concept of chaotic transport in terms of flux across partial barriers on the unstable manifolds has been developed to study the particle escape from one region of phase space to another $[3,4]$. This transport theory was originally developed for Hamiltonian systems with two degrees (or one and half degrees) of freedom. Its mathematical framework has been generalized to Hamiltonian systems with multidegrees of freedom [5] and successfully applied to a four-dimensional symplectic map [6]. These important developments in nonlinear dynamics should play a role in the study of beam particle loss caused by tune modulation. In fact, as we will show below, transport theory provides an effective tool to investigate the effect of tune modulations on the betatron motion when a single resonance is dominant. The parameter dependence of the particle loss rate obtained with transport theory agrees well with the results of the multiparticle tracking study and the results of the beam study experiments.

Consider a two-dimensional symplectic map $\left(x_{n+1}, p_{n+1}\right)=T\left(x_{n}, p_{n}\right)$, where $x_{n}$ and $p_{n}$ are the phase-space coordinate and its conjugate momentum after $n$ iterations, respectively. For any phase-space region which contains a fixed point and is bounded by a closed curve, the flux across the boundary is defined as the area occupied by all phase-space points mapped from the interior to the exterior of this region in one iteration of map $T$ and the particle escape from the region can be characterized by the flux across the boundary. Even though the evaluation of the flux across an arbitrary boundary in phase space is not known, the flux across a resonance can be evaluated numerically. Consider an $m$ th-order resonance which has $m$ pairs of elliptic and hyperbolic points. A phase-space boundary on the resonance can be constructed in the following way [7]. First, connect two neighboring hyperbolic points with an arbitrary curve $C_{0}$ and draw $C_{0}$ through the elliptic point between these two hyperbolic points (see Fig. 1). Iterating $C_{0}$ once with $T$, one obtains $T C_{0}$ which again connects two hyperbolic points and goes through an elliptic point. If we iterate $C_{0} m-1$ times, the result is a closed curve $C=\left\{C_{0}, T C_{0}, T^{2} C_{0}, \ldots, T^{m-1} C_{0}\right\}$ which connects all the elliptic and hyperbolic points of the resonance and separates the phase space into two parts. Now iterating $C$ once more, we have another closed curve $C^{\prime}=\left\{T C_{0}, T^{2} C_{0}, \ldots, T^{m-1} C_{0}, T^{m} C_{0}\right\}$. All parts of $C^{\prime}$ coincide with $C$ except the last segment $T^{m} C_{0}$ which connects the original two hyperbolic points on $C_{0}$. Except in a nongeneric integrable case, $T^{m} C_{0}$ and $C_{0}$ typically intersect only at three points on the periodic orbits since otherwise more than two periodic orbits would exist on $C_{0}$. This yields the structure in Fig. 1, which is called the turnstile [4]. The area in one lobe of the turnstile is the area escaping through $C$ on each iterate of the map and therefore is the flux. By area preservation, the two lobes have the same area. Such construction of

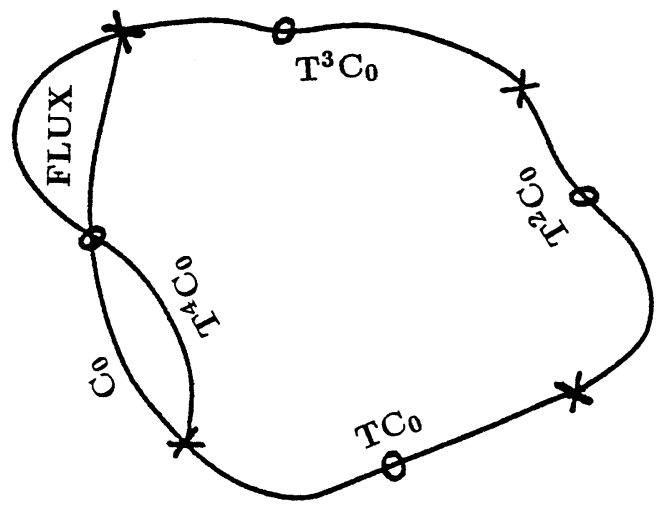

FIG. 1. Formation of the phase-space boundary with turnstile on a 4 th-order resonance. $X$ indicates hyperbolic orbit and $O$ elliptic. 
a boundary has localized the entire flux to one region, and the flux can simply be calculated by evaluating the area of one lobe. For twist maps, this calculation can be accomplished in terms of generating functions $[3,4]$. A map is a twist map if $\partial x_{n+1} / \partial p_{n} \neq 0$ for all $x_{n}$ and $p_{n}$. For a twist map, there exists a generating function $L\left(x_{n}, x_{n+1}\right)$ such that $p_{n}=-\partial L\left(x_{n}, x_{n+1}\right) / \partial x_{n}$ and $p_{n+1}=\partial L\left(x_{n}, x_{n+1}\right) / \partial x_{n+1}$. It can be shown $[3,4]$ that the area in one lobe can be calculated as

$$
\text { Flux }=W\left(\left\{e_{i}\right\}\right)-W\left(\left\{h_{i}\right\}\right),
$$

where $\left\{e_{i} \mid i=0, \ldots, m-1\right\}$ with $e_{m}=e_{0}$ and $\left\{h_{i} \mid i=\right.$ $0, \ldots, m-1\}$ with $h_{m}=h_{0}$ denote the phase-space coordinates for the elliptic and hyperbolic points, respectively, and $W\left(\left\{x_{i}\right\}\right)=\sum_{i=0}^{m-1} L\left(x_{i}, x_{i+1}\right)$ is the action of orbit $\left\{x_{i} \mid i=0, \ldots, m-1\right\}$. The flux across $C$ is thus given by the difference of action between the elliptic and hyperbolic orbits. This is independent of the way $C$ is constructed and, therefore, uniquely defines the flux through a resonance which is, in fact, the flux across the stable and unstable manifolds of hyperbolic points.

In a charge-particle storage ring, the betatron motion in the horizontal plane can be described by a two-dimensional one-turn map. Since the combination of any linear element with any nonlinear element in the thin-lens approximation can be expressed as a symplectic twist map, the generating function for the one-turn map can be obtained as a sum of the generating functions for the combined elements and the flux across a resonance can be calculated in terms of Eq. (1). The number of betatron oscillations for a particle traveling one turn around the ring is called the betatron tune which is denoted as $\nu$. Because of the power supply ripple, ground motion, or synchrobetatron coupling, $\nu$ is modulated with time. The modulation of the tune can usually be modeled by

$$
\nu(n)=\nu_{0}+\delta \nu \sin (2 \pi n \omega),
$$

where $\nu_{0}, \delta \nu$, and $\omega$ are the designed betatron tune, modulation amplitude, and modulation frequency, respectively. $n$ is the number of turns that the particle has circled around the ring. Because of modulation, the one-turn map for the betatron motion becomes time dependent. If the modulation frequency is a rational number, i.e., $\omega=l / k$ where $l$ and $k$ are relatively prime integers, the $k$-turn map is still a time-independent map. To study the effect of the tune modulation, one can first consider the system without the modulation. After choosing a resonance in the phase-space region of interest, flux across the resonance can be calculated with Eq. (1). When the tune is modulated, periodic orbits of the one-turn map are no longer periodic, but as long as the modulation is weak, the $k$-turn map still possesses the same resonance in the same phase-space region. Flux across the resonance of the $k$-turn map contains information of additional particle escape caused by tune modulation and can be evaluated with the periodic orbits of the $k$-turn map. The effect of tune modulation can thus be studied with a comparison between flux for the system with modulation and that without modulation.
Let $\Delta D_{m}(1,0)$ denote the flux across an $m$ th-order resonance in the phase-space region of interest for the system without the modulation and $\Delta D_{m}(k, \delta \nu)$ denote flux across the same resonance of the $k$-turn map when the tune is modulated with a modulation frequency $\omega=$ $l / k$. We conjecture that the effect of modulation can be characterized by

$$
\zeta=\Delta D_{m}(k, \delta \nu) / k-\Delta D_{m}(1,0),
$$

where $\Delta D_{m}(k, \delta \nu) / k$ is the average flux per turn when the tune is modulated and $\zeta$ is the increment of the flux induced by tune modulation. It should be noted that even though the dominant resonance in the phase-space region of interest should be the first candidate for calculating $\zeta$, the correlation between $\zeta$ and the order of resonances needs to be studied in detail. Since an irrational number can be approximated by a sequence of rational numbers, the effect of tune modulation with an irrational frequency can be studied by successively studying modulations with rational frequencies.

As an example, we study tune modulation on a ring with one sextupole kick and otherwise linear. The oneturn map for the betatron motion in the horizontal plane can be written as a time-dependent Henon map,

$$
\left\{\begin{array}{l}
x_{n+1}=\cos [\mu(n)] x_{n}+\sin [\mu(n)]\left(p_{n}-x_{n}^{2}\right), \\
p_{n+1}=-\sin [\mu(n)] x_{n}+\cos [\mu(n)]\left(p_{n}-x_{n}^{2}\right),
\end{array}\right.
$$

where $\mu(n)=2 \pi \nu(n)$ and $\nu(n)$ is given in Eq. (2). Figure 2(a) shows the phase-space portrait of map (4) without tune modulation $(\delta \nu=0)$ as $\nu_{0}=0.2114$. The dynamic aperture (stable boundary) is located between the 21 st-order resonance and the 5th-order resonance which is the dominant resonance limiting the dynamic aperture. The orbits started inside the dynamic aperture stay inside for a long time. In order to have a general picture of the phase space of the modulated system, we plotted, in Figs. 2(b) and 2(c), the phase-space portraits of map (4) and its 537-turn map when the tune is modulated as $\omega=1 / 537$ and $\delta \nu=10^{-3}$. It can be seen that some orbits started inside the original dynamic aperture become unstable when the tune is modulated. Figure 2(c) shows that the multiturn map of a weakly modulated system possesses a similar resonance structure as that of the original system without the modulation.

Without tune modulation, $\nu=\nu_{0}$ and the action for a period- $m$ orbit of map (4) is

$$
\begin{aligned}
W_{m}^{(1)}\left(\left\{x_{i}\right\}\right)=\sum_{n=0}^{m-1}\left\{\frac { 1 } { 2 \operatorname { s i n } ( \mu ) } \left[\cos (\mu)\left(x_{n+1}^{2}+x_{n}^{2}\right)\right.\right. \\
\left.\left.-2 x_{n+1} x_{n}\right]-\frac{1}{3} x_{n}^{3}\right\} .
\end{aligned}
$$

When tune is modulated with $\omega=l / k$, the action for a period- $m$ orbit of the $k$-turn map is

$$
\begin{gathered}
W_{m}^{(k)}\left(\left\{x_{i}\right\}\right)=\sum_{i=0}^{(k m)-1}\left\{\frac { 1 } { 2 \operatorname { s i n } ( \mu ( i ) ) } \left\{\cos [\mu(i)]\left(x_{i+1}^{2}+x_{i}^{2}\right)\right.\right. \\
\left.\left.-2 x_{i+1} x_{i}\right]-\frac{1}{3} x_{i}^{3}\right\}
\end{gathered}
$$



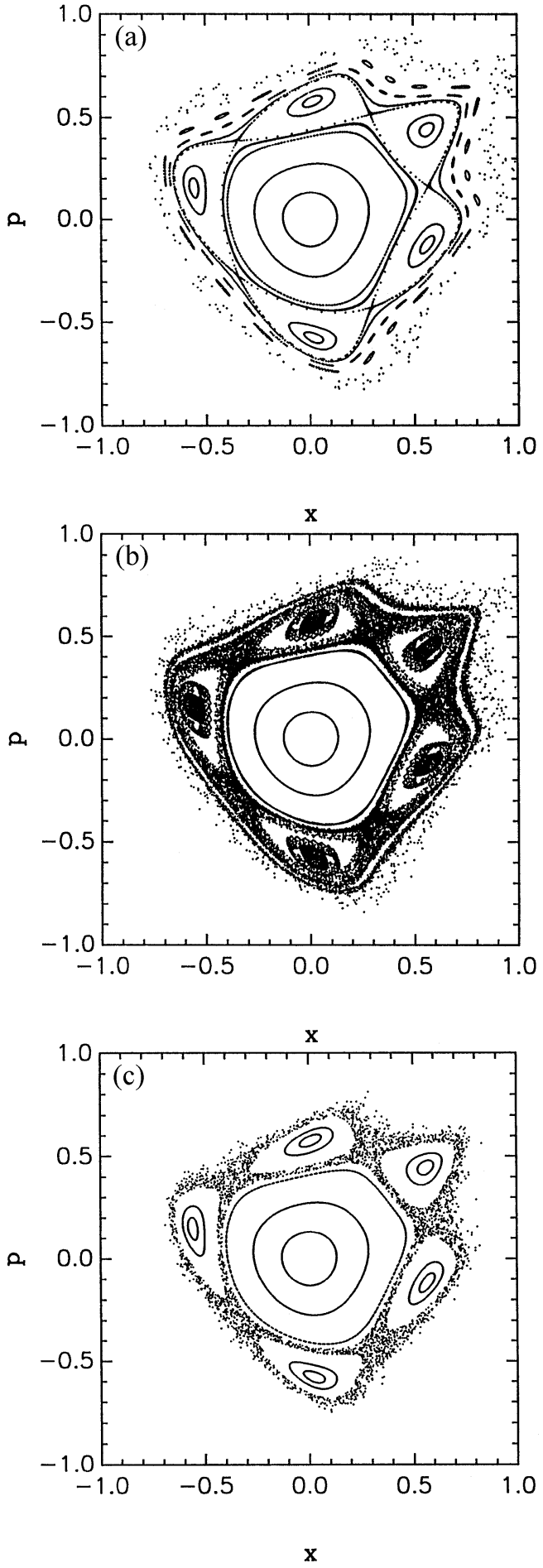

FIG. 2. Phase-space portraits of map (4) when $\nu_{0}=0.2114$. (a) Tune is not modulated $(\delta \nu=0)$; (b) tune is modulated with $\omega=1 / 537$ and $\delta \nu=10^{-3}$; and (c) phase-space portrait of 537-turn map when tune is modulated as (b).

Evaluating the flux across a resonance by using Eqs. (1) and (5) or (6) requires the knowledge of the phasespace coordinates of the elliptic and hyperbolic points. For the one-turn map, a search for the elliptic and hyperbolic points can be accomplished by using the Newton method with the map. The hyperbolic orbit has a positive Lyapunov exponent and the Lyapunov exponent of the hyperbolic orbit of the $k$-turn map is about $k$ times larger than that of the one-turn map. A very large positive Lyapunov exponent implies a very fast exponential growth of numerical errors so that the method of the iteration of the map cannot be used on the $k$-turn map. To locate highly unstable periodic orbits, one has to solve the Lagrange equations for the orbits. The details of the computation of the periodic orbits can be found in Ref. [8].

In order to examine the effect of the modulation when $\omega$ is irrational, $\zeta$ was calculated on the fifth-order resonance when $\omega$ approached irrational numbers with sequences of continued-fraction approximations [8]. It was found that when $\omega$ approaches the irrational number, $\zeta$ converges very fast and no singularity was observed. This suggests that the effect of tune modulation can be understood by only considering rational modulation frequencies.

In Fig. $3, \zeta$ calculated on the fifth-order resonance is plotted as a function of modulation frequency for different modulation amplitude as $\nu_{0}=0.2114$. The calculation on higher-order resonances nearby the fifth-order resonance reveals a similar amplitude and frequency dependence of $\zeta$. Figure 3 shows that the particle loss rate due to tune modulation strongly depends on the modulation amplitude but weakly depends on the modulation frequency. This phenomenon has been observed in the CERN Super Proton Synchrotron (SPS) experiments [1]. The effect of tune modulation increases more than linearly with the modulation amplitude as it was confirmed by the experiments [1]. In Table I, a comparison is given between the amplitude dependence of $\zeta$ and the amplitude dependence of the particle loss rate measured in the SPS experiments when the dominant resonance is fifth order [1]. As $\delta \nu$ is increased from $1.1 \times 10^{-3}$ to $1.65 \times 10^{-3}$, both $\zeta$ and the loss rate are doubled. The particle loss due to tune modulation is therefore well characterized by $\zeta$ when the modulation is weak. As $\delta \nu$ is further increased to $2.2 \times 10^{-3}$, the particle loss rate is increased by a factor of 10 while $\zeta$ is only increased by a factor of 4 . This discrepancy could be due to the additional particle escape along the vertical plane in the experiments when the modulation is strong. Even though flat beams were used and the linear coupling was well corrected in the experiments, particle escape along the vertical plane can be significant when the modulation is strong as it was observed in the experiments [1]. $\zeta$ here, however, only characterizes particle escape along the horizontal plane.

To further examine transport theory, a multiparticle tracking study has been conducted [8] with an ensemble of initial phase-space points chosen within a band covering a phase-space region containing the fifth-order resonance and bounded by the 21 st-order resonance. Let $N_{n}(\delta \nu)$ and $N_{n}(0)$ denote the number of points escaped after $n$ iterations of map (4) with and without tune modulation, 


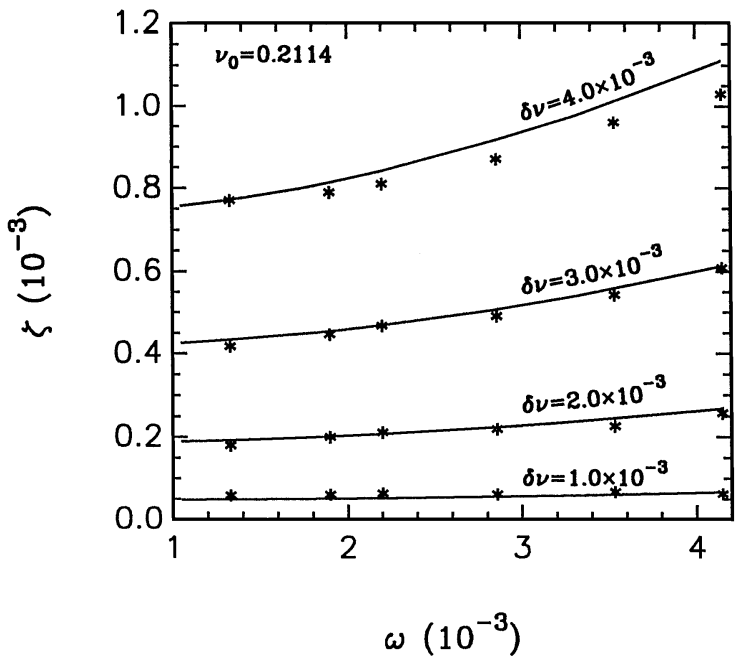

FIG. 3. $\zeta$ as a function of $\omega$ for different $\delta \nu$ when $\nu_{0}=$ 0.2114 (solid curves). Asterisk points are the scaled rate of the particle loss due to the modulation estimated from the multiparticle tracking. The scaling was done in such a way that the loss rate equals $\zeta$ at $\omega=1 / 241$ and $\delta \nu=10^{-3}$. The points plotted along each solid curve are for the same $\delta \nu$ as that of the curve which is indicated on the curve.

respectively. $\left[N_{n}(\delta \nu)-N_{n}(0)\right] / n$ is the average of the escape due to tune modulation in $n$ iterations. It should be noted that $n$, the number of iterations, should be larger than the period of tune modulation but not be too large since, as a large number of points have been lost, points remaining in the ensemble are too few to give a significant statistical result. When $n$ is several hundred of the modulation period, we found that $\zeta \sim\left[N_{n}(\delta \nu)-N_{n}(0)\right] / n$. In Fig. 3 , the scaled $\left[N_{n}(\delta \nu)-N_{n}(0)\right] / n$ is plotted as asterisk points where the scaling has been done in such a way that $\left[N_{n}(\delta \nu)-N_{n}(0)\right] / n$ equals $\zeta$ at $\omega=1 / 241$ and $\delta \nu=10^{-3}$. (This point for the scaling is arbitrarily chosen with relatively weak modulation since the tracking result and the flux-calculation result are expected to agree with each other best there.) Very good agreement was found between the result of the flux calculation and the result of the multiparticle tracking.

This work shows that the concept of chaotic transport in terms of flux across resonances is very useful to the understanding of particle escape due to tune modulation. It provides a computationally efficient means of study-

TABLE I. A comparison of the $\delta \nu$ dependence of the particle loss rate measured in the SPS experiments and $\zeta . D_{1.1}$ and $\zeta_{1.1}$ are the values of the loss rate and $\zeta$ at $\delta \nu=1.1 \times 10^{-3}$, respectively.

\begin{tabular}{ccc}
\hline \hline$\delta \nu\left(10^{-3}\right)$ & Loss rate & $\zeta$ \\
\hline 1.1 & $D_{1.1}$ & $\zeta_{1.1}$ \\
1.65 & $2 \times D_{1.1}$ & $2 \times \zeta_{1.1}$ \\
2.2 & $10 \times D_{1.1}$ & $4 \times \zeta_{1.1}$ \\
\hline \hline
\end{tabular}

ing particle escape from single resonances or particle loss along the horizontal plane when tune is weakly modulated $\left(\delta \nu \ll \nu_{0}\right.$ and $\left.\omega \ll \nu_{0}\right)$. In the case of strong modulation, the resonance structure of the system is severely distorted and, consequently, particle escape cannot be well characterized by $\zeta$ and the calculation of $\zeta$ will also become too difficult to be practical. This, however, appears to not be a problem for tune modulation in storage rings since most cases there involve only weak tune modulation. In order to have a complete understanding of the effect of tune modulation on the betatron motion in storage rings especially in proton machines, particle escape, however, needs to be investigated in the four-dimensional transverse phase space. To extend transport theory to the four-dimensional case, the challenge is to evaluate the flux on a three-dimensional manifold (manifolds of codimensional one) which partitions the four-dimensional phase space into disjoint regions. It was shown that single resonances may possess the manifolds of codimensional one, the three-dimensional stable and unstable manifolds of a two-dimensional normally hyperbolic invariant manifold (NHIM), and the flux across the resonance can be evaluated if NHIM can be located [5]. The difficulty here is a lack of the general algorithm for computation of arbitrary NHIMs except for some special cases [6]. A thorough study of the properties of NHIMs in accelerator models is therefore needed.

The author is indebted to the European Organization for Nuclear Research where this work was begun. $\mathrm{He}$ thanks Dr. J. Gareyte and Dr. W. Scandale for their advice and warm hospitalities. He also thanks Professor S. Ohnuma for encouraging his study of this problem and for many stimulating discussions. This work is supported by the U.S. Department of Energy Grant No. DE-FG0394ER40853.

[1] W. Fischer et al., in Proceedings of the 1993 IEEE Particle Accelerator Conference (IEEE, New York, 1993), p. 246; in Proceedings of the 4th European Particle Accelerator Conference, edited by V. Suller and Ch. PetitJean-Gonaz (World Scientific, Singapore, 1994), p. 950.

[2] O. Bruning and F. Willeke, in Proceedings of the 4th European Particle Accelerator Conference (Ref. [1]), p. 991.

[3] D. Bensimon and L.P. Kadanoff, Physica (Amsterdam) 13D, 82 (1984).

[4] R. MacKay, J.D. Meiss, and I.C. Percival, Physica (Amsterdam) 13D, 55 (1984).

[5] S. Wiggins, Chaotic Transport in Dynamical Systems (Springer-Verlag, Berlin, 1992).

[6] R.E. Gillilan and G. S. Ezra, J. Chem. Phys. 94, 2648 (1991).

[7] J. D. Meiss, Part. Accel. 17, 9 (1985).

[8] J. Shi (to be published). 